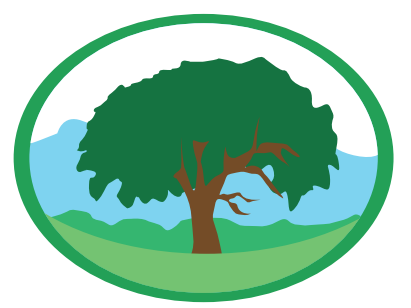

\title{
TEXTURA DO SOLO: CONCEITOS E APLICAC̣ÕES EM SOLOS ARENOSOS
}

\author{
CENTENO, L.N ${ }^{1}$, GUEVARA, M.D.F. ${ }^{2}$, CECCONELLO, S.T. ${ }^{3}$, SOUSA, R.O.D. ${ }^{4}$, TIMM, L.C. ${ }^{5}$ \\ 1 Mestranda em Pós-graduação em Recursos Hídricos na Universidade Federal de Pelotas \\ ${ }^{2}$ Mestrando Programa de pós-graduação em Manejo e Conservação do Solo e da Água \\ ${ }^{3}$ Mestranda no PPG em Ciências Ambientais/Universidade Federal de Pelotas \\ ${ }^{4}$ Professor titular, Departamento de Solos, FAEM-UFPEL
}

${ }^{5}$ Docente do PPG em Recursos Hídricos e do PPG em Manejo e Conservação do Solo e da Água

Palavras-chave: usos e manejo, granulometria do solo, tipo de cultura.

\section{Resumo}

A textura do solo é um dos principais parâmetros utilizados como indicadores de qualidade física do solo, através da sua determinação é possível inferir sobre outros parâmetros, além de compreender o comportamento e manejo do solo, importantes para a produtividade agrícola. Porém, o emprego deste termo vem sendo utilizado de maneira equivocada, uma vez que ao avaliar a textura isoladamente, diversos autores a associam a produtividade do solo. Ciente disto, este trabalho objetivou elaborar uma revisão bibliográfica sobre a influência da textura do solo arenoso na produtividade agrícola, através da compilação de diversos estudos de casos em diferentes cenários. Onde foi possível observar através da classificação de 12 texturas distintas de solo que a produtividade dos solos, principalmente de textura arenosa, não está apenas relacionada as porções de argila, silte e areia, deste solo, mas sim, com o tipo de cultura, o manejo empregado, a localização deste solo frente a disponibilidade hídrica dentre outros fatores. Concluiu-se, portanto, que os solos arenosos podem ser utilizados para o cultivo, desde que seja realizada a implantação de sistemas de produção que consista em novos arranjos e combinações de espécies. Porém, para que isso ocorra é necessária uma gestão do uso e manejo, que considere a textura de cada tipo de solo, bem como das necessidades de diferentes culturas.

\section{SOIL TEXTURE: CONCEPTS AND APPLICATIONS IN SANDY SOILS}

Keywords: uses and management, granulometry soil, type of culture.

\begin{abstract}
The soil texture is a main parameters used as indicators of physical soil quality, through its determination it can infer other parameters, in addition to understanding the behavior and soil management, important for agricultural productivity, but use of this term has been used wrongly, since to evaluate the texture forthwith, various authors, several authors associate it with soil productivity. So, this work aimed to elaborate a bibliographical review on the influence of sandy soil texture on agricultural productivity, through the compilation of several case studies in different scenarios. It was possible to see, through the classification of 12 distinct soil textures, that the soil productivity, mainly of sandy texture, is not only related to the clay, silt and sand portions of this soil, but to the type of management, the location of this soil in front of the water availability among other factors. It was concluded, therefore, that the sandy soils can be used for the cultivation, since the implantation of systems of production is made that consists of new arrangements and combinations of species. However, for this to occur, management of the use and management is necessary, considering the texture of each type of soil, as well as the needs of different cultures.
\end{abstract}




\section{INTRODUÇ̃̃o}

Atualmente os termos textura e granulometria estão sendo empregados como sinônimo em diversos estudos. O que é um fato preocupante, pois textura é uma das características do solo relacionada às partículas primárias (areia, silte e argila) e a sensação que estas partículas oferecem ao tato (atrito, sedosidade e pegajosidade) (BERTONI; LOMBARDI NETO, 2012). Por conseguinte, granulometria do solo está relacionada às porcentagens dessas partículas primárias, distribuídas em diferentes tamanhos definidos por diâmetros específicos (KLEIN, 2014).

A textura, é um dos principais indicadores de qualidade e produtividade dos solos (WANG et al., 2005), uma vez que influencia na dinâmica da adesão e coesão entre as partículas de solo bem como o manejo dos solos, que, por conseguinte influencia a resistência do solo à tração bem como a dinâmica da água no solo. Além disto, pode ser usado como fator ambiental, pois influencia diretamente nos processos ecológicos, tais como a ciclagem de nutrientes e troca de íons (HE et al., 2014).

Este parâmetro físico apresenta uma concepção padrão atual baseada no princípio de que um solo para ser considerado produtivo deve apresentar uma textura argilosa (SANTOS et al., 2003a). Este paradigma se originou no passado, pois solos de textura arenosa eram de pouca relevância para a agricultura, decorrente das suas limitaçóes de manejo, como: deficiência em fertilidade, alta susceptibilidade à erosão, contaminação das águas subterrâneas e deficiência hídrica quando em sequeiro (RAMALHO FILHO; BEEK, 1995).

É pertinente ressaltar ainda que segundo Donagemma et al. (2016), solos arenosos, são mais propensos a degradação e à perda da capacidade produtiva quando comparados aos solos argilosos, em condiçóes ambientais similares; este fato aumenta a sustentação em relação aos dogmas criados para com os solos arenosos. Porém, esta concepção vem mudando atualmente, em função dos avanços nos sistemas de produção e manejo, tornando viáveis as atividades agrícolas nesses solos.

Diante disto o objetivo deste estudo foi elaborar uma revisão bibliográfica sobre a influência da textura do solo arenoso na produtividade agrícola, através da compilação de diversos estudos de casos em diferentes cenários.

\section{MATERIAL E MÉTODOS}

\section{-CARACTERIZAC̣ÃO DOS SOLOS DE ACORDO COM A TEXTURA}

Antes de realizar a classificação textural de um determinado solo, de acordo com o teor de argila, silte e areia, é necessário identificar a distribuição dessas partículas no solo, para então ser possível determinar o tipo de textura através do emprego dos triângulos texturais (KLEIN, 2014).

Com a determinação da textura do solo é possível obter uma estimativa indireta de diversos fatores dentre eles: a dinâmica da água, resistência do solo a tração, grau de compactação do solo, capacidade de troca de cátions, dosagem de nutrientes, corretivos e de herbicidas. Cabe ressaltar que a textura do solo não é sujeita a mudanças de classe, sendo esta uma característica intrínseca do solo, podendo assim ser somente afetada quando ocorrer à mistura com solos de texturas diferentes.

Dentro da classificação textural existem três grupos principais de classes conhecidas, sendo estas: solos arenosos, francos e argilosos; sendo que em cada grupo coexistem as classes texturais específicas, que totalizam 13 classes texturais. Estas classes fornecem uma ideia da distribuição do tamanho de partículas e indicam o comportamento das propriedades físicas do solo (BRADY; WEIL, 2013).

Observa-se que os solos com textura arenosa apresentaram maiores deficiências de fósforo e matéria orgânica de acordo com a Tabela 1. Isto ocorre, porque estes solos apresentam em média $70 \%$ de sua composiçáo teores de areia, o que os tornam altamente permeáveis, com baixa capacidade de retenção de água, baixos teores de matéria orgânica e adsorção de íons. Frente a isso se faz necessário uma gestão do uso e manejo adequado para estes solos, bem como, quando possível, investir na reposição da matéria orgânica do solo e nas práticas conservacionistas; para torná-los menos suscetíveis à erosão e aumentar sua aptidão agrícola (BRADY; WEIL, 2013).

Solos de textura franca, também conhecidos como textura média, definem-se como solo com proporçóes 
semelhantes de partículas de areia, silte e argila; o que os torna com boa drenagem e capacidade de retenção de água e índice médio de erodibilidade.

As características dos solos francos encontrados na Tabela 1, mostram que a maioria dos solos do tipo franco arenosos tendem a apresentar deficiências tanto em matéria orgânica como de fósforo, enquanto os de textura franco argilosa possuem maiores teores desses parâmetros. De forma geral, estes tipos de solos necessitam de poucos cuidados durante seu manejo quando comparado aos solos de textura arenosa

Tabela 1: Compilação de estudos de casos que relacionaram a textura do solo com a produtividade

\begin{tabular}{|c|c|c|c|c|c|c|c|c|c|c|}
\hline \multirow{3}{*}{ AUTOR E ANO } & \multirow{3}{*}{ LOCAL } & \multirow{3}{*}{ TEXTURA } & \multicolumn{3}{|c|}{ GRANULOMETRIA } & \multirow{2}{*}{ MO } & \multirow{3}{*}{ CULTURA } & \multirow{3}{*}{$\mathrm{pH}$} & \multirow{3}{*}{$\frac{\text { CTC }}{\text { Cmole } \mathrm{dm}^{-3}}$} & \multirow{3}{*}{$\begin{array}{c}\mathrm{P} \\
\mathrm{mg} \mathrm{dm^{-3 }}\end{array}$} \\
\hline & & & Areia & Silte & Argila & & & & & \\
\hline & & & \multicolumn{4}{|c|}{. } & & & & \\
\hline \multirow{3}{*}{ SANTOS et al. (2003a) } & \multirow{3}{*}{ Mato Grosso do Sul } & Arenosa & & & 80 & 9,8 & \multirow{3}{*}{ Soja } & 4,8 & 4,45 & 43,0 \\
\hline & & Franca & & & 253 & 15,5 & & 5,2 & 7,43 & 16,8 \\
\hline & & Argilosa & & & 644 & 30,4 & & 5,3 & 13,34 & 12,2 \\
\hline \multirow{6}{*}{$\begin{array}{c}\text { VINHAL-FREITAS } \\
\text { (2013) }\end{array}$} & \multirow{6}{*}{ Minas Gerais } & \multirow{3}{*}{ Muito argilosa } & 111 & 108 & 780 & 60,5 & Cerrado & 4,3 & 12,90 & 1,3 \\
\hline & & & 111 & 97 & 792 & 47,4 & Pastagem & 5,5 & 7,80 & 1,0 \\
\hline & & & 117 & 148 & 732 & 35,5 & Cana-de-açúcar & 6,6 & 6,00 & 11,5 \\
\hline & & \multirow{3}{*}{ Franco argijo-arenosa } & 649 & 147 & 225 & 35,3 & Cerrado & 4,9 & 7,20 & 0,9 \\
\hline & & & 627 & 169 & 224 & 28,6 & Pastagem & 5,6 & 4,80 & 2,6 \\
\hline & & & 642 & 167 & 260 & 25 & Cana-de-açúcar & 5,7 & 4,60 & 12,2 \\
\hline AKUNE (2015) & Vale do Ribeira (SP) & Arcilo-giltogat & 409 & 430 & 160 & 26,8 & Milho & 5,4 & 15,52 & 28,2 \\
\hline \multirow{2}{*}{$\begin{array}{l}\text { WERLE, GARCIA e } \\
\text { ROOSOLEN (2008) }\end{array}$} & \multirow{2}{*}{ Botucatu (SP) } & Franca & & & 80 & 15,0 & \multirow{2}{*}{ Soja } & 4,3 & 6,20 & 25,0 \\
\hline & & Argilosa & & & 253 & 24,0 & & 4,5 & 10,80 & 26,0 \\
\hline \multirow{3}{*}{$\begin{array}{l}\text { PEGORARO et al. } \\
\text { (2008) }\end{array}$} & \multirow{3}{*}{ Belo Horizonte (MG) } & Arenosa & 880 & 30 & 90 & 16,0 & \multirow{3}{*}{ Soja } & 5,8 & & \\
\hline & & Franco argilo-arenosa & 620 & 80 & 300 & 10,0 & & 6 & & \\
\hline & & Argilosa & 250 & 110 & 640 & 71,0 & & 5,5 & & \\
\hline \multirow{2}{*}{ BRAUN et al. (2009) } & \multirow{2}{*}{ Alegre (ES) } & Argilosa & 400 & 530 & 70 & & \multirow{2}{*}{ Café } & 6,2 & 4,20 & 12,0 \\
\hline & & Argilo-arenoga & 651 & 310 & 39 & & & 6,1 & 3,10 & 7,0 \\
\hline $\begin{array}{c}\text { FERREIRA, } \\
\text { CLEMENT e } \\
\text { RANZANI (1989) }\end{array}$ & Manaus (AM) & Franco argilo-arenosa & 671 & 21 & 308 & 12,9 & Pupunheira & 4,7 & 1,85 & 4,0 \\
\hline \multirow{2}{*}{ תUNIOR et al (2008) } & \multirow{2}{*}{ Іаиақаси (PR) } & Franco arenosa & 880 & 20 & 100 & & \multirow{2}{*}{ Capim } & 5,4 & 4,05 & 7,9 \\
\hline & & Argilosa & 330 & 90 & 560 & 16,0 & & 5,6 & 12,18 & 33,9 \\
\hline \multirow{2}{*}{$\begin{array}{c}\text { GONÇAL VES, } \\
\text { DEMATTE e COUTO } \\
(1990)\end{array}$} & \multirow{2}{*}{ São Paulo } & Muito argilosa & 894 & 34 & 71 & 23,0 & & 3,8 & 4,85 & 2,1 \\
\hline & & Franco argilo-arenosa & 536 & 185 & 238 & 33,0 & Eucalipto & 4,3 & 10,65 & 10,8 \\
\hline
\end{tabular}

MO: Matéria Orgânica; pH: Potencial Hidrogâñigniç: P: Fósforo 
Continuação da Tabela 1: Compilação de estudos de casos que relacionaram a textura do solo com a produtividade de culturas

\begin{tabular}{|c|c|c|c|c|c|c|c|c|c|c|}
\hline \multirow{3}{*}{ AUTOR E ANO } & \multirow{3}{*}{ LOCAL } & \multirow{3}{*}{ TEXTURA } & \multicolumn{3}{|c|}{ GRANULOMETRIA } & \multirow{2}{*}{ MO } & \multirow{3}{*}{ CULTURA } & \multirow{3}{*}{$\mathrm{pH}$} & \multirow{3}{*}{$\begin{array}{c}\text { CTC } \\
\text { Comold dm }\end{array}$} & \multirow{3}{*}{$\frac{\mathrm{P}}{\mathrm{mg} \mathrm{dm^{-3 }}}$} \\
\hline & & & Areia & Silte & Argila & & & & & \\
\hline & & & \multicolumn{4}{|c|}{$\ldots$} & & & & \\
\hline \multirow{2}{*}{ NUNES (2016) } & \multirow{2}{*}{$\begin{array}{l}\text { Formoso do } \\
\text { Araguaia (TO) }\end{array}$} & Areia franca & 790 & 12 & 198 & 12,0 & \multirow{2}{*}{ Arroz } & 4,6 & 4,38 & 206 \\
\hline & & Argila arenosa & 615 & 13 & 372 & 39,0 & & 4,9 & 8,68 & 63,4 \\
\hline \multirow{2}{*}{ DOU et al (2016) } & \multirow{2}{*}{ Texas (U.S.) } & Muito argiloga & 140 & 220 & 640 & 12,0 & \multirow{2}{*}{ Arroz } & 5,9 & & 13 \\
\hline & & Franco arenosa & 690 & 160 & 150 & 7,0 & & 5,9 & & 36 \\
\hline \multirow{2}{*}{ JAREMTXHUK et al. (2009) } & \multirow{2}{*}{ Maringá (PR) } & Franco arenosa & 700 & 160 & 140 & 7,6 & \multirow{2}{*}{ Múltiplas } & 5,8 & 5,39 & 9,4 \\
\hline & & Muito argiloga & 150 & 20 & 830 & 11,2 & & 7,8 & 13,96 & 3,0 \\
\hline \multirow{2}{*}{ HE et al (2014) } & \multirow{2}{*}{ Canadá } & Franco siltosa & 314 & 504 & 182 & & \multirow{2}{*}{ Trigo } & & & \\
\hline & & Argila & 50 & 360 & 590 & & & & & \\
\hline \multirow{3}{*}{$\begin{array}{l}\text { OYINLOLA e JINADU } \\
\qquad(2012)\end{array}$} & \multirow{3}{*}{ Nigéria } & Areia & 880 & 100 & 20 & 2,2 & \multirow{3}{*}{ Tomate } & 4 & 5,10 & 3,2 \\
\hline & & Franca & 400 & 360 & 240 & 7,2 & & 4,5 & 4,60 & 4,5 \\
\hline & & Argila & 280 & 240 & 480 & 7,9 & & 4,2 & 7,60 & 5,4 \\
\hline \multirow{4}{*}{$\begin{array}{c}\text { GANDHI e PALIWAL } \\
(1976)\end{array}$} & \multirow{4}{*}{ India } & Franco argilosa & 360 & 280 & 322 & 8,0 & \multirow{4}{*}{ Trigo } & 8,2 & 34,80 & \\
\hline & & Franco argilo-arenosa & 538 & 190 & 221 & 6,7 & & 8,2 & 15,00 & \\
\hline & & Franco arenosa & 725 & 45 & 180 & 5,6 & & 8,3 & 9,80 & \\
\hline & & Areia franca & 805 & 75 & 115 & 3,2 & & 8,3 & 8,20 & \\
\hline \multirow{4}{*}{ CAMBOURIS et al. (2016) } & & Argila giltosa & 62 & 498 & 440 & 30,9 & & 7,1 & 25,73 & \\
\hline & & Franca & 458 & 373 & 169 & 26,3 & & 7,2 & 14,13 & \\
\hline & Quebec (Canada) & Franco arenosa & 664 & 231 & 105 & 19,6 & Milho & 7,5 & 10,93 & \\
\hline & & Areia franca & 837 & 103 & 60 & 50,3 & & 5,8 & 11,90 & \\
\hline & & Arango argilo-5iltoga & 181 & 464 & 355 & 18 & Miscanthuse & 8,3 & 22,30 & 69,3 \\
\hline RONCUCCl et al. (2015) & Pisa (ltälia) & Franco arenosa & 789 & 110 & 101 & 9 & Banicum & 7,7 & 8,50 & 116,9 \\
\hline & & Franco arenosa & 650 & 260 & 90 & 75,9 & & 6,0 & & 8,7 \\
\hline ZHAO et al. (2015) & Quingdar(China) & Franco giltogat & 230 & 580 & 190 & 132.6 & Amendoim & 6,1 & & 10,1 \\
\hline & & Argilo siltosa & 90 & 460 & 450 & 157,4 & & 5,9 & & 9,6 \\
\hline & & Franco giltosa & 253 & 503 & 244 & 11,4 & & 4,9 & 28,60 & \\
\hline $\operatorname{SMITH}(1970)$ & lowa (E.U.A) & Muito argiloga & 0 & 145 & 855 & 12,6 & Pinus gyblieatras & 5,4 & 4,80 & \\
\hline
\end{tabular}

MO: Matéria Orgânica; pH: Potencial Hidtogâñioniçs: P: Fósforo.

Por fim os solos argilosos apresentam um manejo difícil, por serem solos mais pesados devido à presença de teores de argila superiores a 35\%, dificultando a penetração das raízes das plantas e os trabalhos mecanizados, além de serem suscetíveis à compactação (KLEIN, 2014).

Na maior parte dos casos é possível observar que a textura do solo argiloso pode influenciar diretamente no teor de matéria orgânica do solo (Tabela 1), estreitamente relacionado com a capacidade de troca de cátions dos respectivos solos afetando o $\mathrm{pH}$, além da adsorção de fósforo bem como de outros nutrientes (BRADY; WEIL, 2013).

\section{POTENCIAL AGRÍCOLA DOS SOLOS}

Os atuais sistemas de manejo do solo tornaram os solos produtivos independente da textura do solo. Este fato pode ser observado no estudo realizado por Santos et al. (2003a), por exemplo, onde o autor revelou que a produtividade da soja apresentou resultados superiores em solos de textura média e arenosa, quando comparados aos argilosos. É importante salientar ainda que o efeito positivo ou negativo do tipo de textura depende ainda das exigências de desenvolvimento do tipo de cultura.

De acordo com Galbieri et al. (2014), no Estado de Mato Grosso, a produtividade de algodão não difere entre solos argilosos e de textura média. Dou et al. (2016), relataram que solos argilosos possuem maior matéria orgânica quando comparados aos solos arenosos, isto ocorre devido à maior proteção física oferecida pela argila, ocorrendo menores perdas de matéria orgânica e consequentemente maior capacidade de água disponível à planta, o que proporcionou neste estudo, um acentuado aumento na produtividade da cultura do arroz.

O Eucalipto é outra cultura que se adequa bem em solos arenosos. O eucalipto é a essência florestal mais cultivada no Brasil, estende-se por aproximadamente 5 milhões de hectares, sendo a maior parte cultivada 
O trabalho de Flores et al. (2010), na regiáo de Alegrete, no Rio Grande do Sul, deixa evidente que o sistema de plantio pode favorecer a qualidade do solo, uma vez que empregado de maneira adequada. Neste estudo os autores empregaram o sistema silvopastoril, com eucalipto e ovinos, em solos arenosos, e observaram um aumento da rentabilidade em comparação à pecuária extensiva.

\section{PRODUTIVIDADE DO SOLO}

4.1.Textura versus Matéria Orgânica e Capacidade de Troca de Cátions

Verificar que o estudo de Zhao et al. (2015) foi o que possuiu dentre todos os estudos compilados, quando se referindo a solos arenosos, a maior quantidade de matéria orgânica (Tabela 1). Este fato pode estar associado ao sistema de plantio empregado. $\mathrm{O}$ fato do sistema de plantio ser relevante para a conservação da matéria orgânica no solo pode ser evidenciado quando comparado o estudo supracitado ao estudo de Santos et al (2003a) que utilizou o sistema convencional.

Porém, no estudo de Santos et al. (2003a) apresentando pouca matéria orgânica no solo arenoso, encontrou resultados satisfatórios de produtividade de soja, fato este similar ao de Zhao et al. (2015) onde a produtividade de amendoim foi baixa em solos de textura argilosa em relação ao solo arenoso com baixo teor de matéria orgânica.

Mas analisar apenas a matéria orgânica $(\mathrm{MO})$ de forma isolada não é suficiente para se ter uma visão global do que mais influenciou, sendo necessário deixar claro que diversos fatores influenciam diretamente na produtividade destas culturas, como por exemplo o próprio tipo de cultura empregado, o clima da regiáo, a disponibilidade de água e nutrientes.

Os estudos de Silva et al. (1994) corroboram com os supracitados, uma vez que os autores avaliando as interaçóes existentes entre matéria orgânica (MO) e a capacidade de troca de cátions (CTC), em dois solos distintos, um Neossolo Quartzarênico e um Latossolo Vermelho-Amarelo de textura média e argilosa respectivamente, sob o cultivo de soja, constataram que a diminuição no teor de $\mathrm{MO}$ foi sincrônico ao decrescimento da CTC em pH 7,0, e também ao período de cultivo.

Com base na Tabela 1 é possível verificar o mesmo relacionamento entre as variáveis $\mathrm{MO}$ e CTC, o que era esperado, pois solos arenosos possuem menor matéria orgânica quando comparados aos demais solos e consequentemente apresentam menor capacidade de troca de cátions, ou seja apresentam baixa capacidade de adsorção de cátions (BRADY; WEIL, 2013). Sendo os maiores valores de $\mathrm{MO}$ encontrados onde o manejo adotado visa a "proteção" do solo, deixando explícito que o manejo influencia o poder tampão e a resiliência dos solos arenosos; o que reafirma o que havia sido supracitado, ou seja, a necessidade de manter a cobertura do solo, bem como quando possível adotar um sistema de plantio de acordo com a realidade do local.

\subsection{Textura versus a Calagem do solo}

A calagem pode tornar os solos arenosos bem mais produtivos uma vez que o calcário neutraliza o alumínio tóxico, ao elevar o $\mathrm{pH}$, e assim liberar o cálcio e magnésio necessário para as plantas. Um fato curioso é que diversos produtores incorporam em solos arenosos dosagem maior do que a recomendação de calcário, principalmente na cultura do algodão, uma vez que os autores relataram que a quantidade adequada resultou em baixa produtividade (BRADY; WEIL, 2013). De acordo com Donagemma et al. (2016) "esta técnica" pode ser explicada pela baixa reatividade do calcário nesses solos, em razão do baixo teor de alumínio e do baixo poder tampão do solo, e por possíveis perdas de cátions em profundidade, pela lixiviação.

\subsection{Textura versus Fósforo}

Ao observar a Tabela 1 é possível verificar que quanto mais arenoso é o solo menor é a quantidade de fósforo nestes solos. A baixa quantidade deste nutriente no solo se deve principalmente a baixa adsorção do fósforo às partículas de areia do solo, e principalmente pela baixa porção de argila e silte nestes solos, o que diminui a capacidade de adsorção bem como a área superficial específica deste solo (KLEIN, 2014).

Este fator pode ser prejudicial ao desenvolvimento das plantas uma vez que este nutriente não se encontra acessível no solo. E outro fator alarmante é que este nutriente em solos arenosos é mais facilmente 
lixiviado, o que pode acarretar em contaminação dos mananciais. Porém, se for realizado o manejo adequado como, por exemplo, a aplicação de fósforo apenas no sulco do plantio, pode minimizar os riscos ambientais (DONAGEMMA et al., 2016).

Uma vantagem em se utilizar de solos arenosos, para produção agrícola, quando comparados aos solos argilosos é que a menor capacidade tampão desses solos, em relação ao fósforo, faz com que diminua a quantidade empregada de calcário para a correção da acidez (BRADY; WEIL, 2013).

Contudo, são necessários novos estudos para se obter uma visão mais singular e detalhada de como as diferentes texturas juntamente com os sistemas agrícolas escolhidos se comportam com a incorporação de fertilizantes e insumos em geral.

Outro fato relevante é que de acordo com Nunes (2016), os teores de nutrientes encontrados na parte aérea da planta foram influenciados pelas texturas do solo e que teve efeito diretamente na produtividade de grãos. De acordo com Nunes (2016) o solo de textura argilo-arenosa, apresentou teores considerados como suficientes, para o desenvolvimento do arroz, já no caso do solo de textura franco arenosa os níveis do nutriente supracitado estavam abaixo do necessário, este fato segundo o autor está relacionado aos baixos teores de matéria orgânica no solo franco arenoso.

\section{AGRADECIMENTOS}

Agradecemos à CAPES (Coordenação de Aperfeiçoamento de Pessoal de Nível Superior) pela concessão da bolsa durante todo esse período de realização da pós-graduação nos PPG em Recursos Hídricos e Manejo e Conservação do Solo e da Água.

\section{CONCLUSÃO}

Com base nesta revisão é possível concluir que a produtividade do solo está totalmente relacionada com a textura deste solo. Porém, esta relação também se encontra envolta de outras características, como por exemplo, o tipo de manejo adotado bem como a cultura empregada.

Contudo, solos arenosos podem ser utilizados para o cultivo, desde que seja realizada a implantação de sistemas de produçáo que consista em novos arranjos e combinaçôes de espécies. Para que isso ocorra é necessária uma gestão adequada do uso e manejo do solo, que considere a textura de cada tipo de solo, bem como das necessidades de cada cultura.

\section{LITERATURA CITADA}

AKUNE, V. S. C. Cultivo de milho verde em sucessão ao arroz no Vale do Ribeira, SP: subsídios para adoção de zonas de manejo. 123f. Universidade Estadual Paulista "Júlio de Mesquita Filho", Dissertação (Mestre em Agronomia), 2015.

BERTONI, J.; LOMBARDI NETO, F. Conservação do solo. 8. ed. São Paulo: Ícone, 2012. 355 p.

BRADY, N.C.; WEIL, R.R. Elementos da natureza e propriedades dos solos. 3. ed. Porto Alegre: Bookman, 2013. 790p.

BRAUN, Heder et al. Desenvolvimento inicial do café conillon (coffea canephora pierre) em solos de diferentes texturas com mudas produzidas en diferentes substratos. Idesia (arica), [s.l.], v. 27, n. 3, p.35-40, dez. 2009. SciELO Comision Nacional de Investigacion Cientifica Y Tecnologica (CONICYT). http:// dx.doi.org/10.4067/s0718-34292009000300006.

CAMBOURIS, A. N.; et al. Corn yield components response to nitrogen fertilizer as a function of soil texture. Canadian Journal Soil Science, v. 96, p. 1-14, 2016. http://dx.doi.org/10.1139/ cjss-2015-0134

DONAGEMMA, Guilherme Kangussu et al. Characterization, agricultural potential, and perspectives for the management of light soils in Brazil. Pesquisa Agropecuária Brasileira, [s.l.], v. 51, n. 9, p.1003-1020, set. 2016. FapUNIFESP (SciELO). http:// dx.doi.org/10.1590/s0100-204x2016000900001.

DOU, F.; et al. Soil Texture and Cultivar Effects on Rice (Oryza sativa, L.) Grain Yield, Yield Components and Water Productivity in Three Water Regimes. PLOSONE, v. 11, p. 1-12, 2016.

FERREIRA, S. A. N.; CLEMENT, C. R.; RANZANI, G. Contribuição para o conhecimento do sistema radicular da pupunheira (Bactris gasipaes H. B. K.- Guilielma gasipaes (H. B. K.) Bailey), Acta Amazonica, v. 10, n. 2, p. 245-249, 1980.

FLORES, C.A.; RIBASKI, J.; MATTE, V.L. Sistema agrossilvipastoril na região sudoeste do estado do Rio Grande do Sul. 2010. Disponível em: <www.infobibos.com/Artigos/2010_4/ SistemaAgroSilvoPastoril/index.htm $>$.

GALBIERI, R.; et al. Áreas de produção de algodão em Mato Grosso: nematoides, murcha de fusarium, sistemas de cultivo, fertilidade e física de solo. Primavera do Leste: IMAMT, 2014. 16p. (Circular técnica IMAMT, 8).

GANDHI, A. P.; PALIWAL, K. V.. Mineralization and gaseous losses of nitrogen from urea and ammonium sulphate in saltaffected soils. Plant And Soil, [s.1.], v. 45, n. 1, p.247-255, ago. 1976. Springer Nature. http://dx.doi.org/10.1007/bf00011146. GONÇALVES, J. L. de M.; DEMATTÊ, J. L. I.; COUTO, H. T. Z. do. Relaçóes entre a produtividade de sítios florestais de eucalyptus grandis e eucalyptus saligna com as propriedades de alguns solos de textura arenosa e media no estado de São Paulo. Instituto de Pesquisa e Estudos Florestais, Piracicaba, v. 44, n. 45, p.24-39, jan. 1990.

HE, Y; et al. A modelling approach to evaluate the long-term effect of soil texture on spring wheat productivity under a rainfed condition. Scientific reports, v. 4, p. 1-12, 2014.

JAREMTCHUK, C.c. et al. Efeito residual de flumioxazin sobre 
a emergência de plantas daninhas em solos de texturas distintas. Planta Daninha, [s.1.], v. 27, n. 1, p.191-196, mar. 2009. Fap UNIFESP (SciELO). http://dx.doi.org/10.1590/s010083582009000100024

KLEIN, V. A. Física do solo. Ed. Universidade de Passo Fundo. $3^{\circ}$ edição, 2014.

LIMA, H.V.; SILVA, A.P.; SANTOS, M.C.; COOPER, M.; ROMERO, R.E. Micromorphology and image analysis of a hardsetting Ultisol (Argissolo) in the state of Ceará (Brazil). Geoderma, v.132, p.416-426, 2006. DOI: 10.1016/j. geoderma.2005.06.006.

NUNES, D. P. Avaliação da Nutrição de Arroz Irrigado Cultivado em Solos com Diferentes Texturas e seu Efeito na Sanidade e Produtividade de Grãos. 86 f. Fundação Universidade Federal do Tocantins, Dissertação (Mestre em Melhoramento Genético Vegetal), 2016.

OLIVEIRA JÚNIOR, Rubem Silverio de et al. Lixiviação e degradação de diuron em dois solos de textura contrastante. Acta Scientiarum. Agronomy, [s.1.], v. 30, n. 5, p.631-638, 12 dez. 2008. Universidade Estadual de Maringa. http://dx.doi. org/10.4025/actasciagron.v30i5.5963.

OYINLOLA, E.Y.; JINADU, S.A.. Growth, Yield and Nutrient Concentrations of Tomato as Affected by Soil Textures and Nitrogen. Asian Journal of Agricultural Research, [s.1.], v. 6, n. 1, p.39-45, 1 jan. 2012. Science Alert. http://dx.doi.org/10.3923/ ajar.2012.39.45.

PEGORARO, F. R.; et al. Crescimento de soja em solos em resposta a doses de boro, calagem e textura do solo. Ciência agrotecnologica, v. 32, n. 4, p. 1092-1098, 2008.

RAMALHO FILHO, A.; BEEK, K.J. Sistema de avaliação da aptidão agrícola das terras. 3.ed. rev. Rio de Janeiro: EMBRAPA CNPS, 1995. 65p.

RONCUCCI, Neri et al. Influence of soil texture and crop management on the productivity of miscanthus (Miscanthus $\times$ giganteusGreef et Deu.) in the Mediterranean. Gcb Bioenergy, [s.1.], v. 7, n. 5, p.998-1008, 3 jun. 2014. WileyBlackwell. http://dx.doi.org/10.1111/gcbb.12202.

aSANTOS, J.Z.L. Fertilizantes fosfatados e produção da soja em solos com diferentes capacidades tampão de fosfato. Revista de Brasileira de Ciência do Solo, v. 27, p. 639-646, 2003.

bSANTOS, Flávia Cristina dos et al. Produtividade e aspectos nutricionais de plantas de soja cultivadas em solos de cerrado com diferentes texturas. Revista Brasileira de Ciência do Solo, [s.l.], v. 32, n. 5, p.2015-2025, out. 2008. FapUNIFESP (SciELO). http://dx.doi.org/10.1590/s0100-06832008000500023.

SILVA, J.E. da; LEMAINSKI, J.; RESCK, D.V.S. Perdas de matéria orgânica e suas relaçôes com a capacidade de troca catiônica em solos da região de cerrados do oeste baiano. Revista Brasileira de Ciência do Solo, v.18, p.541-547, 1994.

SMITH, David William. The effects of soil texture and soil moisture on photosynthesis, growth and nitrogen uptake of scotch pine seedlings. 1970. 70 f. Dissertação (Mestrado) - Curso de Agricultura, Iowa State University, Si, 1970.

STAPE, J.L.; et al. The Brazil eucalyptus potential productivity project: influence of water, nutrients and stand uniformity on wood production. Forest Ecology and Management, v.259, p.1684-1694, 2010. DOI: 10.1016/j.foreco.2010.01.012.

VINHAL-FREITAS, I. C. Matéria orgânica, fluxo de CO2 e índice Q10 em dois latossolos com texturas contrastantes sob diferentes usos no cerrado. 105f. Universidade Federal de Uberlândia, Tese (Doutora em Agronomia), 2013.
WANG, Q.; OTSUBO, K. \& ICHINOSE, T. Digital map sets for evaluation of land productivity. Disponível em: <http://www. iscgm.org/html4/pdf/forum2000/DrQinxueWang.pdf>

WERLE, R.; GARCIA, R. A.; ROSOLEM, C. A. Lixiviação de potássio em função da textura e da disponibilidade do nutriente no solo. Revista Brasileira de Ciência do Solo, v. 32, p. $2297-$ 2505, 2008

ZHAO, C. X.; JIA, L. H.; WANG, Y. F.; WANG, M. L.; MCGIFFEN JR, M. E. Effects of Different Soil Texture on Peanut Growth and Development. Communications in Soil Science and Plant Analysis, v. 46, p. 2249-2257, 2015. 